\title{
Overview of aging research using nonhuman primate models
}

\author{
Ilhem Messaoudi • Donald K. Ingram
}

Published online: 30 December 2011

(C) American Aging Association 2011

The human population is aging at an unprecedented rate. The World Health Organization is predicting that by 2050 , two billion people will be at least 60 years of age (http://www.who.int/ageing/en/). Despite considerable advances in the medical field, age-related health problems continue to seriously compromise quality of life and pose a significant challenge to the health care system. According to the Center for Disease Control, $80 \%$ of individuals aged 65 years and older have at least one chronic disease (www.cdc.gov/chronicdisease/ resources/publications/AAG/aging.htm). In addition, infectious diseases such as tuberculosis, pneumonia, and influenza remain among the ten leading causes of mortality in the USA. Therefore, the development of preventive measures and interventions that can slow

I. Messaoudi

Vaccine and Gene Therapy Institute, Oregon Health and Science University, Portland, OR, USA

I. Messaoudi $(\square)$

Division of Pathobiology and Immunology,

Oregon National Primate Research Center,

Beaverton, OR, USA

e-mail:messaoud@ohsu.edu

D. K. Ingram

Nutritional Neuroscience and Aging Laboratory,

Pennington Medical Research Center,

Louisiana State University System,

Baton Rouge, LA, USA aging or shorten the associated period of deterioration and disease would improve the quality of life for millions while vastly reducing health care costs. Progress towards this goal is heavily dependent on appropriate animal models in which to study the underlying causal mechanisms and test such interventions.

Traditionally, most of our understanding of the biological changes that occur with aging has come from studies with rodents. Rodent models offer several distinct research advantages: short life span, inexpensive husbandry, abundant reagents, and a highly tractable model of genetic manipulation. However, rodents and humans diverged some $84-121 \mathrm{Ma}$ ago, a phenomenon that is likely to result in several fundamental differences between the rodent and human aging processes. One of the major differences is that aged mice do not suffer from several age-related chronic diseases, such as atherosclerosis or diabetes, which are important causes of morbidity among older humans.

Nonhuman primates (NHPs), on the other hand, share significant genetic, physiological, and behavioral traits with humans and have emerged as a leading translational model. Indeed, comorbidity patterns in aging monkeys closely mirror those seen in older humans. For instance, aged macaques experience perturbed sleep-wake cycles and cognitive decline, macular degeneration, loss of motor skills and skeletal mass as well as diabetes, hypertension, osteoporosis, and atherosclerosis. These diseases only appear in rodents with genetic manipulation 
and do not always recapitulate the clinical picture. Furthermore, NHPs are susceptible to either human pathogens or simian pathogens that bear significant homology to human infectious agents and reproduce characteristics and functional sequelae of diseases seen in humans. Moreover, NHPs can be maintained in carefully controlled environments, and similar techniques can be used to assess functional changes facilitating comparisons between NHPs and humans. In addition, NHPs are outbred, which enables rigorous validation of research findings that goes beyond the proof of principle provided by rodent models while avoiding self-selection bias often seen in clinical trials. Finally, because macaques are a long-lived species, it is likely that these NHPs have adapted similar maintenance strategies as humans, making them an ideal translational model.

NHPs used in biomedical research include apes, Old World monkeys (e.g., macaques), and New World monkeys (e.g., marmosets and squirrel monkeys). Evolutionarily, chimpanzees and bonobos (the nearest living relatives of humans) would most faithfully represent human aging. However, practical and ethical considerations, such as their status as endangered species and their long life span (as much as 60 years in captivity), make chimpanzees and other apes less appealing for aging research. The New World monkeys are more phylogenetically distant from Homo sapiens, diverging over $30 \mathrm{Ma}$ ago, whereas Old World monkeys diverged $15 \mathrm{Ma}$ ago. Thus, the rhesus macaque (Macaca mulatta) represents the preferred monkey resource for biomedical research (used in $>70 \%$ of published studies) and has served as an invaluable model for human infectious diseases. However, New World monkeys present practical advantages such as smaller size, ease of handling, and reduced risk of zoonotic disease transmission, which may increase use of these species in the future.

The articles in this special issue of $A G E$ provide an excellent overview of the use of NHPs in biogerontology ranging from cognitive and physical decline to neuroendocrine perturbations and reduced immune function. Dr. Lawrence Sherman and colleagues review the studies in NHPs that support the hypothesis that disturbances in brain white matter start at middle age and that these disturbances contribute to age-related cognitive decline. They provide evidence that these changes may occur as a result of free radical damage, degenerative changes in cells in the oligodendrocyte lineage, as well as changes in microenvironments. Dr. John Morrison and colleagues review studies showing that rhesus monkeys are vulnerable to age-related decline in executive function and memory similar to humans, thereby providing a valuable model for cognitive aging. They summarize age-related changes in anatomical, neuronal, and synaptic parameters within brain areas implicated in cognition and discuss how these changes are associated with cognitive decline. Drs. Caleb Finch and Steven Austad provide an evolutionary perspective into brain aging and discuss the important differences in the rate of cognitive decline across several NHP species. They note that New World macaques may prove especially valuable in the area of Alzheimer's disease research.

Dr. Ricki Colman and colleagues examined the effects of long-term, moderate caloric restriction on skeletal parameters in rhesus monkeys. They report that as described for humans, bone mass and density decline over time with generally higher levels in control compared to calorically restricted animals. Dr. Carol Shively and colleagues examined indices of physical mobility such as gait speed in young and old macaques. They report that self-motivated walking speed was slower in older macaques. Likewise, young adults climbed, leaped, and jumped more frequently than older adults. Of all the behaviors evaluated, walking speed measured in a simple and inexpensive manner appeared most sensitive to age, making it a useful indicator of decline in physical mobility in NHP models of aging.

Dr. Henryk Urbanski and colleagues highlighted some of the most dramatic rhythmic neuroendocrine changes that occur in primates during aging, focusing on changes in male rhesus monkeys. They report that the age-associated attenuation of hormone levels and reduction of circadian signaling are potentially compounded by changes in intracrine-processing enzymes and hormone receptors levels. These observations can guide future studies aimed at improving neuroendocrine function in the elderly. Dr. James Herndon and colleagues provide the first estimates of age-specific rates of menopause in chimpanzees. This finding revealed that female chimpanzees could remain reproductively viable for a greater proportion of their life span than women. Thus, while menopause marks the end of the chimpanzee's life span, women may thrive for decades more.

Dr. Christopher Coe and colleagues examined the impact of aging on the immune response to influenza 
vaccination and the utility of studying aged macaques in order to uncover potential correlates of protective immunity in the elderly. Along these lines, Dr. Christine Meyer and colleagues review the state of various interventions aimed at slowing aging of the immune system that have shown great promise in rodent models and how evaluating these interventions in aged macaques can provide a powerful translational tool.
The authors of the papers contained herein have provided an outstanding overview of the value of NHP in modeling human aging and age-related disease, both the strengths and challenges they present. Beyond this important review, however, the overarching intent of this Special Issue is to educate and interest other researchers so that collaborations might be fostered to further advance the field. 